\title{
Diseño y construcción de un prototipo de superficies equipotenciales como proceso de enseñanza investigativa
}

Guillermo Terán Acosta

Universidad Central del Ecuador gteran30@hotmail.com

Vanesa Chaluiza Charro

Universidad Central del Ecuador vanesa_lt@hotmail.com

Recibido: 01 de septiembre / Aprobado: 30 de noviembre 2017

\section{Resumen}

El presente trabajo tiene como propósito fundamental el diseño y construcción de un prototipo orientado al análisis e inferencia de principios, leyes y aplicaciones en el estudio de las superficies equipotenciales. Para cumplir con dicho propósito se fundamentó teóricamente en el aprendizaje de la física experimental como investigación conducente a la construcción de nuevos conocimientos en el contexto de la resolución de problemas abiertos (Gil. D); así como la conceptualización de campos constantes y superficies equipotenciales a partir del planteamiento de un cuerpo de hipótesis como: el vector campo eléctrico $\boldsymbol{E}$ tiene en cada punto la dirección de la normal a la superficie equipotencial que pasa por dicho punto y, durante la traslación en un segmento $\boldsymbol{d} \boldsymbol{l}$ por un plano equipotencial, el potencial no varía. En este trabajo se describen los elementos estructurales y funcionales del prototipo, con también la utilización de materiales de calidad y bajo costo; describiendo los mate- 
riales utilizados y las precauciones que se requieren para su construcción. Además, se especifican los fundamentos teórico-experimentales para las diferentes aplicaciones del prototipo como herramienta didáctico-pedagógica en los campos de la física. La metodología se apoyó en una investigación de tipo experimental. Los hallazgos experimentales obtenidos se encuentran en los porcentajes de error a razón del 4,64\% en la variación inversamente proporcional del potencial respecto a la distancia.

Palabras clave: superficies equipotenciales, campos constantes, herramientas didáctico pedagógicas, física experimental.

\begin{abstract}
This research aims to show how fundamental it is to design and construct of a prototype oriented for the analysis and inference of principles, laws and applications in the study of equipotential surfaces. To comply with this purpose the theory based on the learning of experimental physics research leading to the construction of new knowledge in the context of the resolution of open problems, (Gil. (D); as well as the conceptualization of constant fields and equipotential surfaces from the approach of a hypothesis as a body: the electric field vector $\mathrm{E}$ has at every point the direction of the normal to the equipotential surface which passes through the point and, during the translation in a segment $\mathrm{dl}$ by an equipotential level, the potential does not change. This paper describes the structural and functional elements of the prototype, with also the use of quality materials and low cost; describing the materials used and the precautions that are required for its construction. In addition, it specifies the pedagogical experimental foundations for different applications of the prototype as a tool in the fields of physics. The methodology relied on experimental research. The experimental findings obtained are in the rate of error of $4.64 \%$ in the inversely proportional variation of the potential with respect to the distance.
\end{abstract}

Keywords: equipotential surfaces, constant fields, pedagogical teaching tools, experimental physics. 


\section{Introducción}

$\mathrm{E}$ n el presente siglo se evidencia una aguda crisis en la educación en todos los niveles y fundamentalmente en la educación superior; la crisis de la educación superior es, entonces, una crisis de cambio, de revisión a fondo de sus propósitos, de sus misiones, de su quehacer, de su organización y métodos de trabajo. Al respecto, Martínez, M. (1999) expresa que la crisis de los paradigmas científicos ha estallado de manera incontenible e inocultable. No solamente estamos en una crisis de los fundamentos del conocimiento científico, sino también del filosófico, y, en general, ante una crisis de los fundamentos del pensamiento.

Corrobora Rivas B. (1998) al plantear que el paradigma tradicional, que ha dominado en los últimos años la teoría y la praxis educativa, ha agotado su potencia y necesita de una nueva concepción.

Estamos en medio de un gran cambio de paradigma. El paradigma se convierte, así, en un principio filosófico direccionador del conocimiento y de la existencia humana, que a decir de Kuhn (1971) en su célebre obra, The estructure of scientific revolutions el paradigma admite pluralidad de significados y diferentes usos: "Es una red de creencias teóricas y metodológicas, valores, ideas, técnicas y procedimientos compartidos por una comunidad científica para solucionar problemas". (p. 13).

El paradigma epistémico (Martínez, M. 1999 y Guba, E. 1990) actual ha ido logrando una serie de metas que pueden formar un conjunto de postulados en donde toda observación es relativa desde el punto de vista del observador, planteados por Einstein; toda observación se hace desde una teoría (Hanson); toda observación afecta al fenómeno observado (Heisenberg); no existen hechos, sólo interpretaciones (Nietzsche); ninguna ciencia está capacitada para demostrar científicamente su propia base (Descartes); ningún sistema matemático puede probar los axiomas en que se basa (Godel); la pregunta ¿qué es ciencia? No tiene respuesta científica (Morín). Estas ideas conforman una plataforma y una base lógica conceptual para asentar todo proceso racional con pretensión científica.

En el contexto actual y, particularmente en el desarrollo académico de las ciencias de la física teórica y experimental, es indispensable definir una sólida postura epistemológica y procedimientos metodológicos pertinentes que permitan orientar la construcción del conocimiento de problemas de la realidad, mediante la investigación científica.

En este contexto, uno de los principales propósitos del trabajo de la física experimental, y coincidiendo con los planteamientos de Salinas (1996), es concebir a las prácticas de laboratorio como investigaciones colectivas en torno a situaciones problémicas que sean de interés de los estudiantes, en las cuales los estudiantes desempeñan el rol de investigadores noveles y, el docente, en el tutor de acompañamiento académico e investigativo que orienta el proceso de modo coherente con un tratamiento científico de la problemática en cuestión, evidenciando así la interrelación entre conceptos, hipótesis, teorías y observaciones. 
Además, desde la perspectiva vygotskiana que hace referencia a la interacción social en los procesos de aprendizaje y de pensamiento (Vygotsky, 1978). Los procesos de pensamiento se originan en actividades mediadas socialmente, principalmente a través del lenguaje. En consecuencia, los estudiantes necesitan oportunidades para aprender ciencias, y particularmente la física teórica y experimental, usando sus ideas y su pensamiento a través de la interacción con su entorno, compañeros y con el docente. Así, el trabajo de laboratorio se concibe como un lugar para el intercambio social, de exploración y expansión de las ideas, apoyados en métodos multidimensionales y multireferenciales de saberes teórico-epistemológicos, saberes metodológico-prácticos y saberes aplicativos crítico-reflexivos; pilares fundamentales para la producción y generación del conocimiento y desarrollo cognitivo.

Al respecto, Tipler, P. (1977) plantea que desde el punto de vista la mecánica newtoniana se pueden realizar diferentes análisis del mundo cuántico con ciertas aproximaciones que permiten estudiar, visualizar y analizar los campos eléctricos y particularmente las superficies equipotenciales, propósito del presente trabajo, considerando que los estudiantes tienen dificultades en la interiorización de esta temática los mismos que interpretan las interacciones electrostáticas, utilizando preferentemente el perfil conceptual coulombiano (Furió y Guisasola, 1998 citado por Del Valle, S., 2016). En este sentido, para el estudio de las superficies equipotenciales se inicia con el diseño, elaboración y construcción del prototipo; constituyéndose en una herramienta didáctica de apoyo en el proceso académico e inferencia de las leyes newtonianas partiendo de un análisis de comportamiento de cada uno de los factores o parámetros físicos que intervienen en este proceso de análisis.

El objetivo central de la investigación es diseñar y construir un modelo (prototipo) desde una perspectiva estructural y funcional, orientado al análisis e inferencia de principios y leyes de las superficies equipotenciales, sobrepasando el modelo de aprendizaje planteado por Gil, D. (1994) de la física experimental como investigación colaborativa, porque se parte del diseño y construcción de los dispositivos para el estudio de este fenómeno, identificando factores y parámetros físicos que conforman el dispositivo, a saber: cuerpos de prueba para la producción de líneas de fuerza de cargas puntuales, planas; diseño de prensas, bandeja prototipos para campos puntuales; lámina milimetrada (serigrafía), entre otros dispositivos, que permiten una participación interactiva entre los docentes y estudiantes. Para lo cual se inicia el proceso académico con el planteamiento de un cuerpo hipótesis de trabajo: a) el vector campo eléctrico $\mathrm{E}$ tiene en cada punto la dirección de la normal a la superficie equipotencial que pasa por dicho punto y b) durante la traslación en un segmento $\mathrm{dl}$ por un plano equipotencial, el potencial no varía.

\section{Relación de magnitudes físicas, intensidad de campo eléctrico y potencial eléctrico}

El campo eléctrico se conceptualiza como una magnitud vectorial $\mathbf{E}$ o de la magnitud escalar $\mathbf{V}$. Entre estas dos magnitudes existe una relación determinada. 
(Savéliev, I., 1982 y Hewitt, P., 1999). Si tomamos en consideración que el campo eléctrico $\mathbf{E}$ es proporcional a la fuerza que actúa sobre la carga y que $\mathbf{V}$ es la energía potencial de dicha carga, en consecuencia esta relación es análoga a la que existe entre la energía potencial y la fuerza.

La fuerza $\mathbf{F}$ está relacionada con la energía potencial por la ecuación:

$$
\mathbf{F}=-\nabla \mathrm{W}_{\mathrm{p}}
$$

Para una partícula cargada que se encuentra en un campo eléctrico, $\mathbf{F}=\mathrm{q} \mathbf{E}_{\mathrm{y}} \mathrm{W}_{\mathrm{p}}=\mathrm{q} \mathbf{V}$. Al reemplazar estos valores en la ecuación (1), obtenemos:

$$
\mathrm{q} \mathbf{E}=-\nabla(\mathrm{q} \mathbf{V})
$$

La constante $q$ se puede sacar del signo del gradiente. Haciendo esto y simplificando después por $q$ llegamos a la ecuación:

$$
\mathbf{E}=-\nabla V
$$

Que determina la relación entre la intensidad de campo eléctrico y el potencial.

Apoyándonos en la definición del gradiente se obtiene la ecuación:

$$
\boldsymbol{E}=-\frac{\partial \boldsymbol{V}}{\partial x} \boldsymbol{e}_{x}-\frac{\partial \boldsymbol{V}}{\partial y} \boldsymbol{e}_{y}-\frac{\partial \boldsymbol{V}}{\partial z} \boldsymbol{e}_{\mathrm{z}}
$$

Por consiguiente, en proyecciones sobre los ejes ortogonales, la relación (3) adquiere la forma:

$$
\begin{aligned}
& \boldsymbol{E x}=-\frac{\partial \boldsymbol{V}}{\partial x} e_{x} \\
& \boldsymbol{E} \boldsymbol{y}=-\frac{\partial \boldsymbol{V}}{\partial y} e_{y} \\
& \boldsymbol{E z}=-\frac{\partial \boldsymbol{V}}{\partial z} e_{z}
\end{aligned}
$$

Por analogía, la proyección del vector campo eléctrico $\mathbf{E}$ sobre una dirección arbitraria $\boldsymbol{l}$ es igual a la derivada de $\mathbf{V}$ respecto de $\boldsymbol{l}$ tomada con signo contrario, es decir, a la velocidad de disminución del potencial durante la traslación a lo largo de la dirección $\boldsymbol{l}$ se obtiene la ecuación: 


$$
\boldsymbol{E}_{\boldsymbol{l}}=-\frac{\partial \boldsymbol{V}}{\partial l}
$$

De que la ecuación (6) es correcta podemos cerciorarnos fácilmente tomando la dirección $\boldsymbol{l}$ como uno de los ejes de coordenadas y teniendo en cuenta la relación (5).

Vamos a explicar la relación (3) valiéndonos del ejemplo del campo de una carga puntual. Pasando las coordenadas cartesianas obtenemos la expresión:

$$
\mathbf{V}=\frac{1}{4 \pi \varepsilon_{\mathrm{o}}} \frac{q}{r}=\frac{1}{4 \pi \varepsilon_{\mathrm{o}}} \frac{q}{\sqrt{x^{2}+y^{2}+z^{2}}}
$$

La derivada parcial de esta función respecto de x es igual a:

$$
\frac{\partial \boldsymbol{V}}{\partial x}=-\frac{q}{4 \pi \varepsilon_{\mathrm{o}}} \frac{x}{\left(x^{2}+y^{2}+z^{2}\right)^{\frac{3}{2}}}=-\frac{q}{4 \pi \varepsilon_{\mathrm{o}}} \frac{x}{r^{3}}
$$

Análogamente

$$
\frac{\partial \boldsymbol{V}}{\partial y}=-\frac{q}{4 \pi \varepsilon_{\mathrm{o}}} \frac{y}{r^{3}}, \quad \frac{\partial \boldsymbol{V}}{\partial z}=-\frac{q}{4 \pi \varepsilon_{\mathrm{o}}} \frac{z}{r^{3}}
$$

Sustituyendo estos valores de las derivadas en la ecuación (4) se obtiene la expresión:

$$
\boldsymbol{E}=\frac{q}{4 \pi \varepsilon_{\mathrm{o}}} \frac{x e_{x}+y e_{y}+z e_{z}}{r^{3}}=\frac{q}{4 \pi \varepsilon_{\mathrm{o}}} \frac{r}{r^{3}}=\frac{1}{4 \pi \varepsilon_{\mathrm{o}}} \frac{q}{r^{2}} e_{\mathrm{r}}
$$

La ecuación (2) permite hallar la intensidad del campo en cada punto por los valores conocidos de V. También puede resolverse el problema inverso, o sea, dado los valores de $\mathbf{E}$ en cada punto, hallar la diferencia de potencial entre dos puntos cualesquiera del campo. Para esto nos valemos de que el trabajo que realizan las fuerzas del campo sobre una carga $\boldsymbol{q}$ al trasladarla desde un punto 1 a un punto 2 se puede calcular como:

$$
A_{12}=\int_{1}^{2} q E d \boldsymbol{l}
$$

Al mismo tiempo, este mismo trabajo se puede representar de la forma

$$
A_{12}=q\left(\mathbf{V}_{1}-\mathbf{V}_{2}\right)
$$


Igualando entre sí estas dos expresiones y simplificando por q, llegamos a la relación

$$
\mathbf{V}_{1}-\mathbf{V}_{2}=\int_{1}^{2} E d \boldsymbol{l}
$$

Esta integral puede tomarse a lo largo de cualquier línea que una los puntos 1 y 2, ya que el trabajo de las fuerzas del campo no depende del camino recorrido. Para el recorrido por un contorno cerrado y la ecuación (7) se transforma en la ecuación:

$$
\oint E d l=0
$$

(la circunferencia en el signo de integral indica que la integración se efectúa a lo largo de un camino cerrado). Advertimos que esta relación solo es válida para el campo eléctrico. Más adelante explicaremos que el campo de las cargas en movimiento (es decir el campo variable con el tiempo) no es de potencial y, por lo tanto, no cumple la condición (8) para éste.

\section{Superficies equipotenciales}

La superficie imaginaria cuyos puntos tienen todos los mismos potenciales eléctricos, se denomina superficie equipotencial.

Su modelo matemático se expresa por:

$$
V(x, y, z)=c t e
$$

Durante la traslación en un segmento $\boldsymbol{d} l$ por un plano equipotencial, el potencial no varía $(\boldsymbol{d} \boldsymbol{V}=\boldsymbol{O})$. Por consiguiente, según la expresión:

$$
E_{l}=-d V / d l=0
$$

El componente del vector $\mathbf{E}$, tangente a la superficie es nulo; implicando que el desplazamiento es perpendicular a una superficie equipotencial.

En el caso del potencial $\mathbf{V}=\mathbf{k} \mathbf{Q} / \mathbf{r}$ producido por una carga puntual $\mathrm{Q}$ en el origen, las superficies definidas por $\boldsymbol{r}=$ constante son superficies equipotenciales. Las equipotenciales son superficies esféricas concéntricas y las líneas de fuerza son en todos los puntos perpendiculares a las superficies equipotenciales.

$\mathrm{Si}$ tenemos un campo eléctrico constante $\mathbf{E}$ producido en el interior de un par de placas paralelas, la diferencia en el valor que toman el potencial eléctrico $\mathbf{V}$ y el campo eléctrico $\mathbf{E}$ para distancias perpendiculares, la variación de dicho potencial eléctrico $\mathbf{V}$ varía en forma proporcional a la distancia. 
Matemáticamente se expresa mediante la ecuación (3)

$$
\mathbf{E}=-\nabla \mathrm{V}
$$

Que determina la relación entre la intensidad del vector campo eléctrico $\mathbf{E}$ y el escalar potencial eléctrico $\mathbf{V}$; y el gradiente definido por el campo $\mathbf{E}$ normal a una superficie.

Cabe mencionar que, en el interior de las placas paralelas, el conjunto de puntos geométricos que se obtienen tienen el mismo valor del potencial. A dichas superficies que cumplen con esas condiciones se las denomina superficies equipotenciales, y las líneas perpendiculares a esa superficie mostrarán la dirección del campo eléctrico.

\section{Materiales y métodos}

La metodología que se utilizó en el presente trabajo se apoyó en una investigación cuantitativa con doble estrategia de una investigación de campo, documental, observacional de carácter descriptivo y de tipo proyecto factible (Terán G. 2006).

El dispositivo de experimentación cuenta con insumos de calidad y bajo costo, a continuación, detallado:

\section{Base de madera}

Se fabricó una base de madera para colocar el recipiente de vidrio, esta base tiene las siguientes medidas:

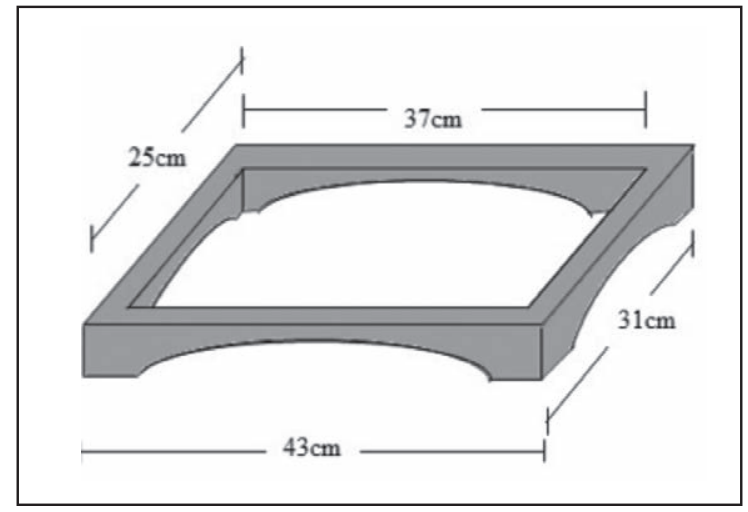

Figura 1. Soporte de madera.

\section{Prensas metálicas}

Las prensas fueron diseñadas en el taller de prototipos del Centro de Física con una lámina metálica de grosor $3 \mathrm{~mm}$, contiene una abertura donde se coloca los cuerpos de prueba, también consta de dos tornillos moleteados para sujetar el cuerpo de prueba con la base de madera. 


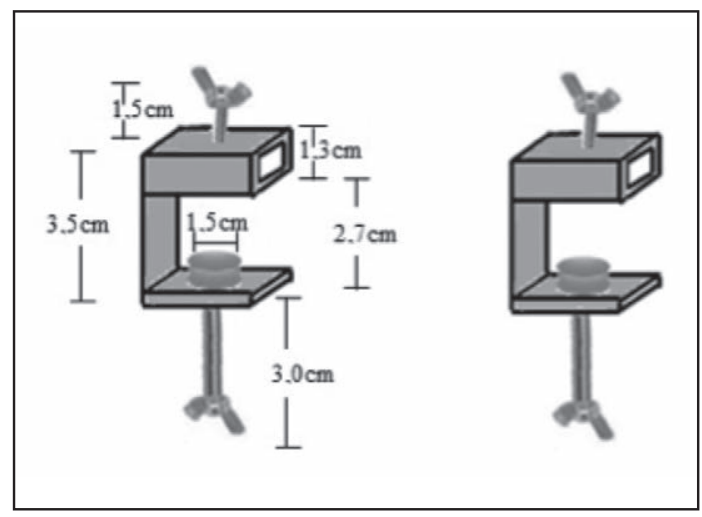

Figura 2. Prensas metálicas.

\section{Cuerpos de prueba}

Para el experimento se diseñaron dos cuerpos de prueba: (a) un par de placas paralelas de $15 \mathrm{~cm}$ de largo y $5 \mathrm{~cm}$ de ancho, para representar campos constantes y, (b) dispositivo de puntas para representar cargas puntuales dos cuerpos de prueba de $15 \mathrm{~cm}$ de largo; los soportes tienen una longitud de $15 \mathrm{~cm}$ y de ancho $1 \mathrm{~cm}$.

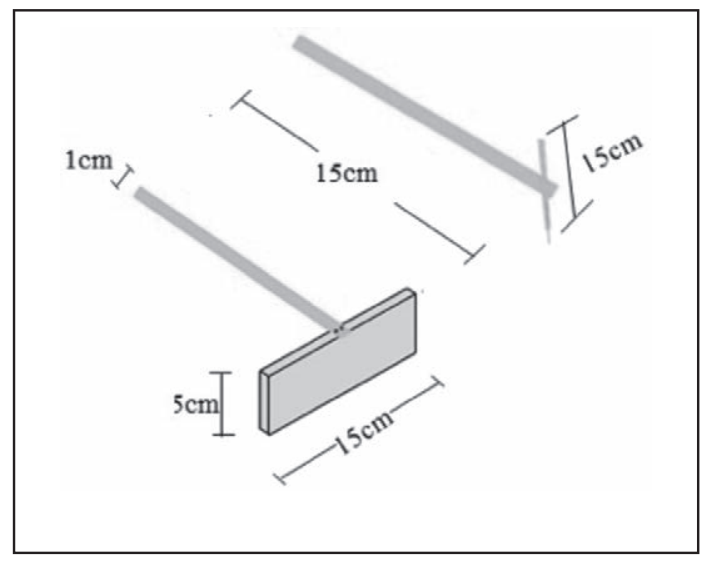

Figura 3. Cuerpos de prueba.

La figura a) es el cuerpo de prueba utilizado para medir los voltajes entre dos cargas paralelas.

La figura b) muestra el cuerpo utilizado para generar el fenómeno de cargas puntuales.

Para el desarrollo de la práctica experimental utilizar dos cuerpos de prueba iguales.

\section{Recipiente de cristal}

El recipiente de vidrio, se consideró en la experimentacion del fenómeno fisico, porque por sus características de estructura molecular no es buen conductor de electricidad. 
Dicho recipiente tiene como dimensiones $35 \mathrm{~cm}$ de largo y $25 \mathrm{~cm}$ de ancho como se representa en la figura 4.

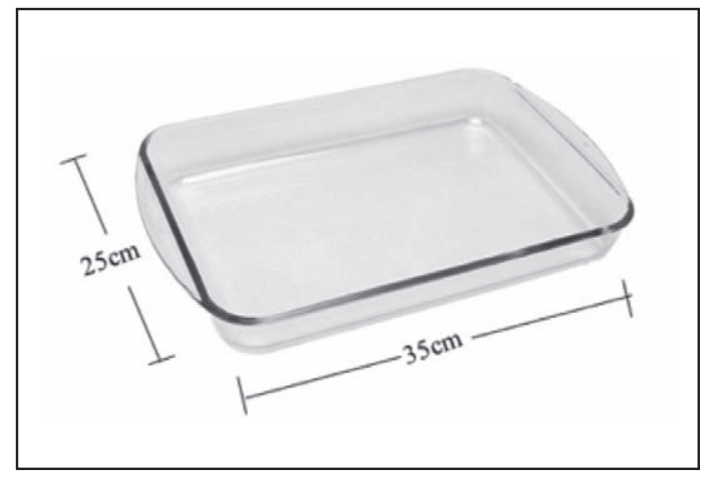

Figura 4. Recipiente de vidrio.

\section{Placa de acrílico}

Esta lámina de $30 \mathrm{~cm}$ de largo y $20 \mathrm{~cm}$ de ancho es serigrafiada en su base con una escala de -15 a 0 y de 0 a 15 , con el propósito de que el estudiante tenga mayor facilidad durante la toma de datos.

Para ayuda de las mediciones en el prototipo se utiliza una hoja de papel milimetrada en acrílico para introducirla dentro del recipiente de cristal y poder colocar agua sobre ella para generar un medio conductor, poder realizar las medidas correspondientes durante el desarrollo de la misma. Con esta placa se puede observar claramente en qué posición se encuentran los voltajes en el plano de coordenadas X y Y.

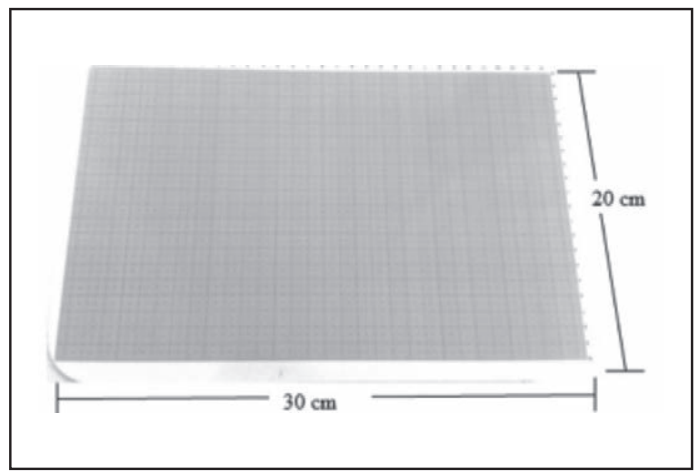

Figura 5. Lámina de acrílico.

Para el desarrollo de la experiencia se debe contar con una fuente de poder de corriente continua, un multímetro de alta precisión, conductores, pinzas lagartos y un recipiente con agua. 
El circuito armado se muestra en la siguiente figura 6.

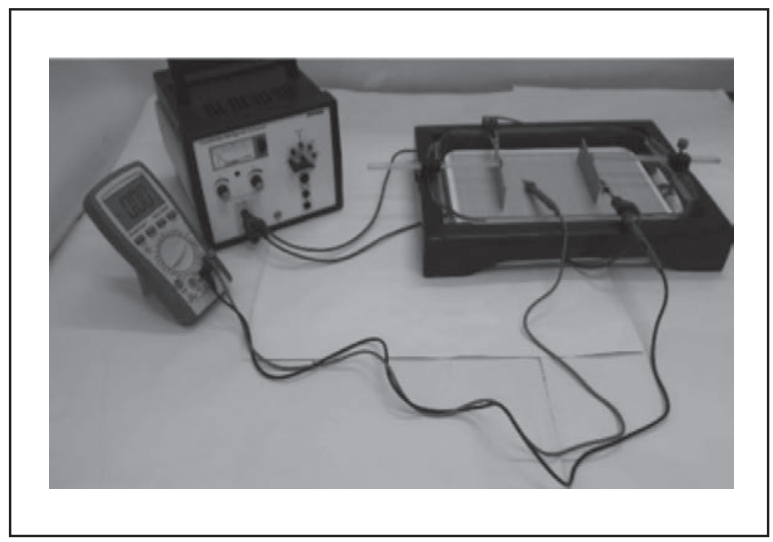

Figura 6. circuito de placas paralelas.

\section{Resultados}

Para la prueba de hipótesis planteadas a) el vector campo eléctrico $\boldsymbol{E}$ tiene en cada punto la dirección de la normal a la superficie equipotencial que pasa por dicho punto y b) durante la traslación en un segmento $\boldsymbol{d l}$ por un plano equipotencial, el potencial no varía, se inició el estudio determinando superficies equipotenciales con dos cargas puntuales y dos placas paralelas, obteniendo los siguientes resultados registrados en la tabla 1, para diferentes voltajes, alrededor de las cargas puntuales $\boldsymbol{Q}^{-}$y $\boldsymbol{Q}^{+}$y entre las placas positiva y negativa respectivamente.

Tabla 1. Resultados de cargas puntuales $Q$ y $\boldsymbol{Q}^{+}$y placas paralelas

\begin{tabular}{|c|c|c|c|c|c|c|c|c|c|c|c|c|c|c|c|c|c|c|c|c|c|}
\hline \multicolumn{12}{|c|}{ Cargas Puntuales } & \multicolumn{10}{|c|}{ Placas Paralelas } \\
\hline \multicolumn{2}{|c|}{$2 \mathrm{~V}$} & \multicolumn{2}{|c|}{$3 \mathrm{~V}$} & \multicolumn{2}{|c|}{$4 \mathrm{~V}$} & \multicolumn{2}{|c|}{$6 \mathrm{~V}$} & \multicolumn{2}{|c|}{$7 \mathrm{~V}$} & \multicolumn{2}{|c|}{$8 \mathrm{~V}$} & \multicolumn{2}{|c|}{$4 \mathrm{~V}$} & \multicolumn{2}{|c|}{$5 \mathrm{~V}$} & \multicolumn{2}{|c|}{$6 \mathrm{~V}$} & \multicolumn{2}{|c|}{$7 \mathrm{~V}$} & \multicolumn{2}{|c|}{$8 \mathrm{~V}$} \\
\hline $\mathrm{X}$ & $\mathrm{Y}$ & $\mathrm{X}$ & $\mathrm{Y}$ & $\mathrm{X}$ & $\mathrm{Y}$ & $\mathrm{X}$ & $\mathrm{Y}$ & $\mathrm{X}$ & $\mathrm{Y}$ & $\mathrm{X}$ & $\mathrm{Y}$ & $\mathrm{X}$ & $\mathrm{Y}$ & $\mathrm{X}$ & $\mathrm{Y}$ & $\mathrm{X}$ & $\mathrm{Y}$ & $\mathrm{X}$ & $\mathrm{Y}$ & $\mathrm{X}$ & $\mathrm{Y}$ \\
\hline$-8,9$ & 0,0 & $-6,7$ & $-0,5$ & $-3,4$ & 0,0 & 3,6 & 0,0 & 6,8 & 0,0 & 9,0 & 0,0 & $-5,3$ & $-3,0$ & $-2,7$ & $-3,0$ & $-0,4$ & $-3,0$ & 2,4 & $-5,0$ & 4,7 & $-4,7$ \\
\hline$-8,7$ & 0,6 & $-7,0$ & $-1,9$ & $-3,6$ & 2,0 & 3,6 & 1,3 & 7,0 & $-0,9$ & 9,2 & 0,9 & $-5,3$ & 1,5 & $-2,7$ & $-2,0$ & $-0,6$ & $-2,0$ & 2,4 & $-4,0$ & 4,7 & $-3,5$ \\
\hline$-9,0$ & 1,2 & $-8,0$ & $-3,5$ & $-3,9$ & 3,0 & 4,4 & 8,2 & 7,1 & $-1,2$ & 10,0 & 1,5 & $-5,3$ & $-1,0$ & $-2,9$ & 1,0 & $-0,4$ & 1,0 & 2,4 & $-3,0$ & 4,7 & $-2,0$ \\
\hline$-9,6$ & 1,5 & $-9,4$ & $-5,6$ & $-4,4$ & 5,5 & 4,0 & 5,0 & 7,4 & $-2,3$ & 9,7 & $-1,5$ & $-5,3$ & 1,0 & $-2,7$ & 4,0 & $-0,5$ & 1,0 & 2,4 & $-2,0$ & 4,7 & $-1,0$ \\
\hline
\end{tabular}




\begin{tabular}{|l|l|l|l|l|l|l|l|l|l|l|l|l|l|l|l|l|l|l|l|l|l|}
\hline$-9,0$ & $-1,0$ & $-6,7$ & $-0,7$ & $-4,4$ & 7,2 & 3,9 & $-3,0$ & 8,4 & $-3,6$ & 9,0 & $-0,7$ & $-5,3$ & $-3,5$ & $-2,7$ & 3,0 & $-0,4$ & 5,0 & 2,4 & $-1,0$ & 4,7 & 1,0 \\
\hline$-10,5$ & 1,0 & $-6,9$ & 2,2 & $-3,8$ & $-2,0$ & 4,3 & $-5,4$ & 7,1 & 1,4 & 9,1 & $-1,0$ & $-5,3$ & 3,0 & $-2,8$ & $-4,0$ & $-0,4$ & $-4,5$ & 2,5 & 5,0 & 4,7 & 2,0 \\
\hline$-10,5$ & $-1,0$ & $-7,4$ & 3,8 & $-4,0$ & $-4,3$ & 4,8 & $-8,4$ & 7,6 & 2,9 & 9,7 & $-1,5$ & $-5,3$ & 4,0 & $-2,7$ & $-2,7$ & $-0,4$ & 1,0 & 2,4 & 3,0 & 4,7 & 3,5 \\
\hline$-9,8$ & $-1,2$ & $-8,2$ & 5,4 & $-4,4$ & $-6,3$ & 4,0 & 5,0 & 8,4 & 4,0 & 10,0 & 1,5 & $-5,4$ & 6,0 & $-2,7$ & $-2,7$ & $-0,4$ & 6,0 & 2,4 & 1,0 & 4,7 & 4,7 \\
\hline
\end{tabular}

Con los datos de la tabla 1 se construyeron las gráficas de las superficies equipotenciales y líneas de campo.

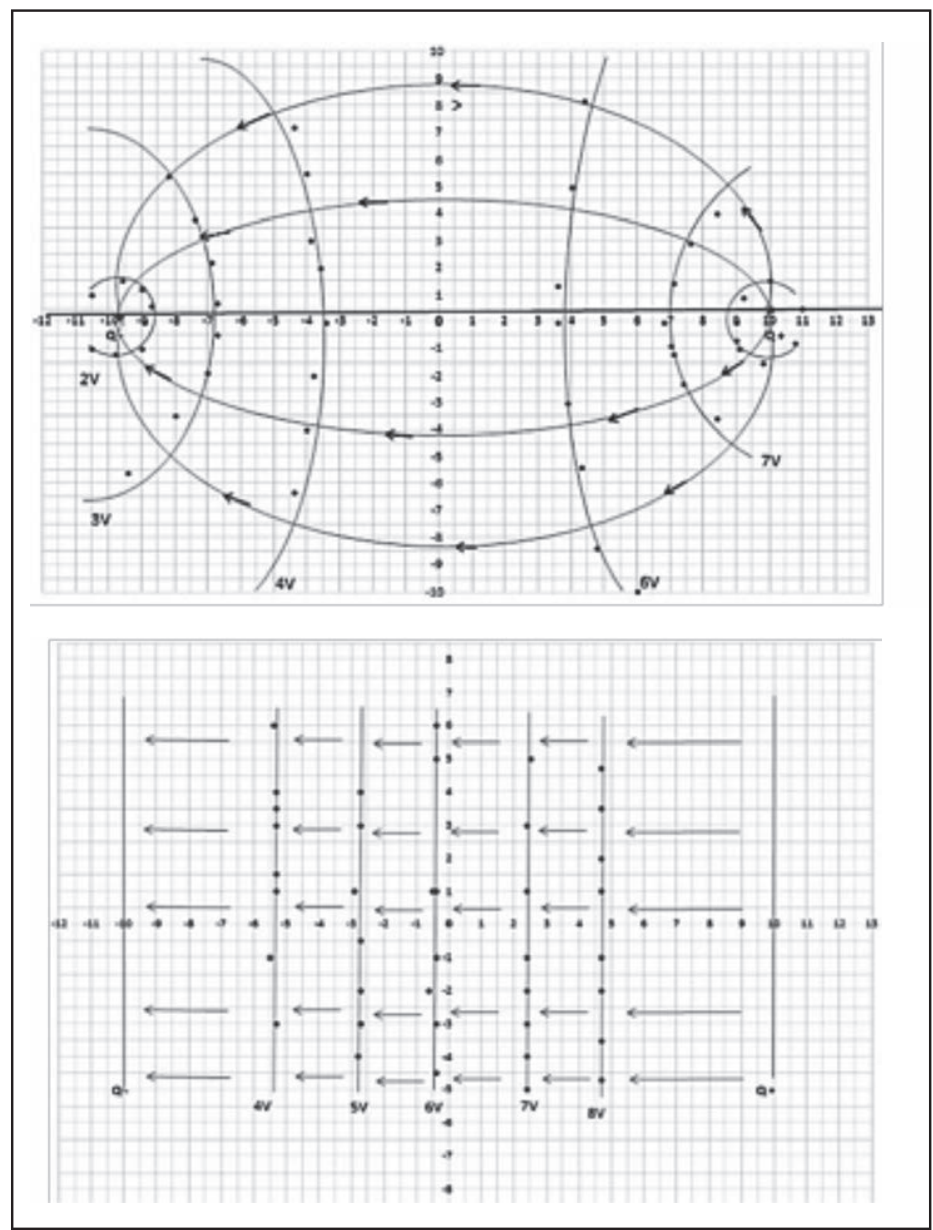

Figura 7. Superfices equipotenciales y líneas de campo de cargas puntuales. 
Además, se determinó el campo eléctrico experimental de placas paralelas, midiendo las distancias entre la carga negativa de la placa y las diferentes líneas equipotenciales formadas debidas a la variación de cada voltaje. También se calcularon los minimos cuadrados para obtener el valor del campo eléctrico con el error respectivo, como se registra en la tabla 2 y figura 8 respectivamente.

Tabla 2. Distancias medidas a cada voltaje y mínimos cuadrados

\begin{tabular}{|c|c|c|c|c|}
\hline \multicolumn{5}{|c|}{ PLACAS PARALELAS } \\
\cline { 1 - 2 } Voltaje & Distancia & \multirow{2}{*}{$\mathbf{y}^{*} \mathbf{y}$} & $\mathbf{x}^{*} \mathbf{x}$ & $\mathbf{y}^{*} \mathbf{x}$ \\
\cline { 1 - 2 }$(\mathbf{V})$ & $(\mathbf{m})$ & & & \\
\hline 0 & 0,000 & 0,000 & 0,000 & 0,000 \\
\hline 4 & 0,053 & 16,000 & 0,003 & 0,212 \\
\hline 5 & 0,076 & 25,000 & 0,006 & 0,380 \\
\hline 6 & 0,104 & 36,000 & 0,011 & 0,624 \\
\hline 7 & 0,153 & 69,000 & 0,016 & 0,889 \\
\hline 10 & 0,200 & 100,000 & 0,040 & 2,000 \\
\hline
\end{tabular}

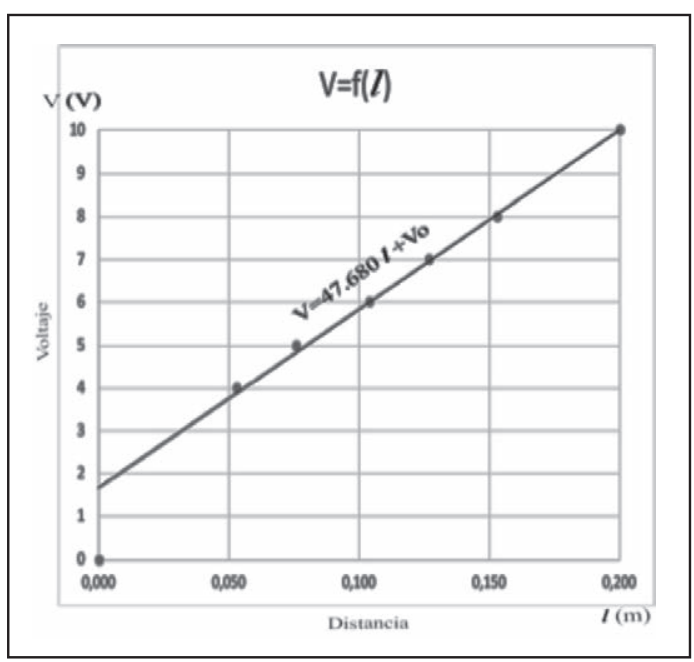

Figura 8. Valor del campo eléctrico. 
Seguidamente se procedió a la construcción gráfica de voltajes versus distancias $\mathrm{V}=\mathrm{f}(l)$; obteniendo la ecuación de la recta con su respectivo error es la siguiente:

$$
K=47,680 \pm 3,490\left(\frac{V}{m}\right)
$$

Con dichos resultados se infiere que la relación de $V=f(l)$, representa una línea recta, en el cual el voltaje varía en forma directamente proporcional a la longitud.

Finalmente se calcula el campo eléctrico, tanto del valor teórico como del experimental, resumido en la tabla 3 .

Tabla 3. Valores de la regresión líneal

\begin{tabular}{|c|c|c|}
\hline Término & Estimación & Error estándar \\
\hline $\begin{array}{c}\text { Constante del } \\
\text { modelo (b) }\end{array}$ & 0,857 & 0,414 \\
\hline Pendiente (m) & 47,680 & 3,490 \\
\hline
\end{tabular}

\section{Cálculo del valor teórico del campo eléctrico}

Valor teórico

$$
\begin{aligned}
& V o=0 \\
& V 1=10 \\
& d=0,20 \mathrm{~m} \\
& E=\frac{V 1-V o}{d} \\
& E=\frac{10}{0,20}=50\left(\frac{V}{m}\right)=50\left(\frac{N}{C}\right)
\end{aligned}
$$

\section{Valor experimental}

$$
\mathrm{E}=47,68\left(\frac{N}{C}\right)
$$

\section{Porcentaje de error}

$$
\% E_{E}=4,64 \%
$$




\section{Conclusiones}

Con base en los hallazgos de la investigación, se estructura un cuerpo estable de ideas conclusivas:

El diseńo y construcción de modelos o prototipos en el proceso de enseñanza y aprendizaje, y particularmente el prototipo para el estudio de fenómenos de superficies equipotenciales, permite una interacción permanente y participativa entre el docente y el estudiante orientada a la construcción de conocimientos.

El aprendizaje de la física experimental debe concebirse como un proceso multidimensional y multireferencial, apoyado en métodos, estrategias y técnicas acordes a los paradigmas de aprendizaje de nueva tendencia, que potencien competencias investigativas y pensamiento crítico y reflexivo de los estudiantes, en el desarrollo de las prácticas de laboratorio.

Mediante el uso del prototipo se probaron las hipótesis planteadas con un margen de error del $\% \mathrm{E}_{\mathrm{E}}=4,64 \%$, para el caso de campos eléctricos producido por un par de placas; donde campo eléctrico $\boldsymbol{E}$ tiene en cada punto la dirección de la normal a la superficie equipotencial que pasa por dicho punto y en el proceso de la traslación en un segmento $\boldsymbol{d} \boldsymbol{l}$ por un plano equipotencial, el potencial no varía.

\section{Referencias}

[1] Del Valle, S. (2016). La experimentación en el aprendizaje de la física. Su incidencia en la construcción de conceptos referidos a la óptica ondulatoria. Tesis doctoral. Universidad Nacional del Centro de la Provincia de Buenos Aires. Facultad de Ciencias Exactas. Núcleo de Investigación en Educación en Ciencia y Tecnología (NIECyT). Tandil.

[2] Gil, D. (1994). Enseñanza de las Ciencias, nro. 11 (2), pp. 197/212. Trabajo presentado como conferencia en la International Conference on History of the Physical-Mathematical Sciences and the teaching of Sciences. Madrid.

[3] Guba, E. (1990). The paradigm dialog. Nueva Delhi. U.S.A.: Sage Publications.

[4] Hewitt, P. (1999). Conceptos de física, edición en español. Limusa Noriega Editores.

[5] Kuhn, T. S. (1971). La estructura de las revoluciones cientificas. México: FCE.

[6] Martínez, M. (1999). La nueva ciencia: Su desafio, lógica y método. México: Editorial Trillas.

[7] Rivas Balboa, C. (1998). Nuevo paradigma para la teoría y praxis educacional. Universidad Simón Bolivar. Caracas, Venezuela: Investigación y Postgrado. Universidad Pedagógica Experimental Libertador.

[8] Salinas, J. (1996). Las prácticas de fisica básica en laboratorios universitarios. Tesis Doctoral. Universitat de València. España.

[9] Savéliev, I. (1982). Curso de física general. Electricidad y magnetismo, ondas, óptica. Moscú: Editorial MIR.

[10]Terán, G. (2006). Hacia una educación de calidad. El proyecto de investigación: Cómo elaborar. Quito-Ecuador. 
[11]Tipler, P. (1977). Física. Barcelona: Editorial Reverté, S. A. (pp. 1098). [12] Vygotsky, L. (1995). Pensamiento y lenguaje. Traducción del original ruso (1978). Ediciones Fausto. Disponible en http://psikolibro.blogspot.com 